\title{
Effectiveness of transcranial direct current stimulation and cognitive therapy based on mindfulness on inhibitory control and selective attention in adults with attention deficient/hyperactivity disorder
}

Niloofar Mikaieli ( $\nabla$ nmikaeili@uma.ac.ir)

University of Mohaghegh Ardabili

Sara moradi kelarde

University of Mohaghegh Ardabili

Mohammad Narimani

University of Mohaghegh Ardabili

Abbasali Hossein Khanzadeh

University of Guilan

Habibeh Salvat

Ardabil university of Medical Science

\section{Research Article}

Keywords: trans-cranial direct current stimulation, mindfulness, inhibition control, selective attention, attention deficit disorder/hyperactivity

Posted Date: February 14th, 2022

DOI: https://doi.org/10.21203/rs.3.rs-1318474/v1

License: (c) (i) This work is licensed under a Creative Commons Attribution 4.0 International License. Read Full License 


\section{Abstract}

Background: Hyperactivity disorder/attention deficit is one of the most adverse neurological disorders that are associated with multiple defects in cognitive and behavioral dimensions, and sometimes continues until adulthood. Today, due to side effects of drugs, compulsion to continue consumption in order to maintain the effects and weak adherence to drug therapy, Non-drug treatments has been welcomed for this disorder. The purpose of this study is to compare the effects of cognitive therapy based on mind awareness and transcranial direct current stimulation on inhibition control and selective attention.

Method: This experimental study has multi-groups pretest-posttest design in which an experimental group is exposed to electrical stimulation intervention ( 5 sessions of 20-minute interventions of electrical stimulation with flow of $1 \mathrm{~mA}$ ) and one another experimental group was exposed to Mindfulness-based cognitive therapy ( 8 sessions 90 minutes, three days a week) and the control group did not receive any intervention. The statistical population is all people with attention deficit/hyperactivity disorder in Rasht. Participants in this study were 45 subjects (15 in each group), which were selected by the convenience method and also in recall.

Results: The results showed that cognitive therapy based on mind awareness and electrical stimulation of the dorsolateral prefrontal cortex had a significant effect on inhibition control and selective attention.

Conclusions: Based on the findings, it is recommended that mindfulness-based cognitive therapy and transcranial direct current stimulation methods be used by psychiatrists, psychologists and psychotherapists in neurological clinics and psychological services as a method of intervention and prevention.

\section{Introduction}

Attention Deficit / Hyperactivity Disorder (ADHD) is a psychological disorder that begins in childhood and results in disproportionate levels of development in the areas of inattention, impulsivity, and hyperactivity; The prevalence of this disorder in children is $3.53 \%$; In many cases, it has been shown to persist into adulthood, and the prevalence of this disorder in adulthood can be $3.4 \%$ (1). This disorder becomes one of the most pervasive developmental neurologic disorders of childhood which showed that it is too current, inattentive or both (2) and with more deficits in cognitive dimensions such as attention and inhibitory control (3). Other cognitive deficits in this dosorde include attention problems, motivational control, and timing (4), indicating that the disorder is in the context of executive functions (5).

Despite the apparent heterogeneity in the neuropsychological formation of Attention Deficit/Hyperactivity Disorder(6), two main models have been hypothesized to explain the behavioral deficits: one, the "model based on inhibition or cognitive impairment." Suggests that inhibition-based executive defects are major defects in the disorder, and lead to failures in executive control that actually lead to impulsive and hyperactive behavior. The "motivational distortion model" emphasizes defective reward processing instead of cognitive deficits, suggesting that behavioral deficits in Attention Deficit /Hyperactivity Disorder result in decreased arousal (7). In other words, poor executive resources (inhibition-based model) and impulse control deficiencies that lead to hyperactivity (motivational dysfunction model) are effective theories in physiological pathology of attention deficit hyperactivity disorder. According to the first model, the decision-making factor leads to incomplete executive functions with hypo-function of the dorso-lateral prefrontal cortex (DLPFC) as well as hyperactivity of the subcortical areas Some executive reactions may play a more fundamental role in the symptoms and defects of this disorder, such as cognitive flexibility, inhibitory control, and selective attention (8).

\section{1 mindfulness-based cognitive therapy and ADHD}

In the treatment of this disorder, drug administration has increased dramatically in recent years. But today, due to the side effects of drugs, the need to continuous use in order to maintain the effects and poor adherence to treatment, non-drug therapies have become very popular. One of the nonpharmacological therapies is mindfulness-based cognitive therapy (MBCT). This therapeutic approach is based on the stress reduction model based on the mindfulness of Kabat-Zin and by adding the principles of cognitive therapy to it. This treatment is a combination of cognitive-behavioral therapy and mindfulness and is a method of teaching sustainable attention. Mindfulness-based cognitive therapy includes attention regulation, emotional regulation, and physical awareness. A research (9) shows that mindfulness has a positive effect on behavioral inhibition. At first glance, mindfulness and ADHD seem to be a contradictory combination; but considering the key aspects of mindfulness makes it clear how useful this method is for improving the symptoms of the disorder. Mindfulness helps the person by informing of what is distracting him or her and helping to restore attention to what was previously focused on, and it can be said that it is in fact a kind of exercise of attention and excitement. Studies also show changes in the brain during mindfulness training that these changes lead to a tendency to a non-invasive stimulation method called direct transcranial electrical stimulation (10).

\subsection{Transcranial direct current stimulation and ADHD}

Recent studies have highlighted the importance of non-invasive brain stimulation in modulating cortical arousal (11, 12). Studies that have examined the modulatory effects of transcranial electrical stimulation on Attention Deficit / Hyperactivity Disorder are rare. Recent studies have highlighted the importance of non-invasive brain stimulation in modulating cortical arousal $(10,11)$. Positive results are reported during the go / do task using cathodic stimulation of the left lateral frontal cortex (13). In a study (14) authors suggested that anodic stimulation in the lower right frontal groove improves inhibitory control. In one study(15), found that cathodic stimulation in the left lateral dorsal forehead area improved inhibitory control in the go / not go 
task, while anodic stimulation in the same area made it difficult. Underlying this effect may be an indirect increase in cortical activity in the right prefrontal cortex, resulting in intracellular communication that enhances inhibitory control.

Barkley has identified behavioral inhibition disorders as the central deficiency of attention deficit/ hyperactivity disorder. Researchers (16) showed that transcranial electrical stimulation improves aspects of selective attention, reducing the time required to select new information and the total number of attention errors.. A study (17) shows that the application of anodic transcranial electrical stimulation to the left DLPFC region helps to improve cognitive function including selective attention, processing speed and working memory.

Neurological studies have shown several aspects of attentional abilities, but the three major parts of attentional abilities that are somehow related to mindfulness are: sustained attention, selective attention, and attention transfer. According to a research (18), correlation coefficient of mindfulness with inhibitory control and selective attention was 0.62 , with a stable focus of 0.44 and with a memory of 0.41 . According to a study (19), mindfulness-based cognitive exercises, in addition to their effect on sustained attention, also improve selective attention capacity. Experimental studies (20) have shown that people who participate in mindfulness exercises have a higher score on the Stroop test (selective attention assessor). About $30 \%$ of adults with the disorder do not respond to standard medication interventions (21). At best, the mediocre effects of behavioral therapy, the high rate of drug withdrawal due to side effects, and the critical attitude of parents toward drug therapy underscore the urgent need to develop alternative therapeutic strategies (22).

\subsection{The present study}

The aim of the present study was to investigate the effects of transcranial electrical stimulation on the lateral dorsal forehead area- which is the most involved area in behavioral inhibition disorders / attention deficit disorder-, as well as mindfulness-based cognitive therapy on inhibitory control and Attention in 45 patients with ADHD in a randomized parallel-group design. Based on previous neuroimaging studies, that showed hypoactivation of prefrontal regions of this area for attention and inhibitory control, we hypothesized that increasing DLPFC activation with anodal tDCS would result in improved inhibition and attention in ADHD (hypothesis 1). Also, according to the literature (9)(23), we hypothesized that MBCT would lead to improved selective attention and inhibition in our experimental group (hypothesis 2).

\section{Materials And Method}

\section{1 participants}

45 patients with ADHD (15 people in the first experimental group, 15 people in the second experimental group and 15 people in the control group) were selected in a targeted and accessible way and also through an online call based on the initial diagnosis of a psychologist, clinical interview Researcher and diagnostic test. Inclusion criteria in this study are the presence of attention deficit / hyperactivity disorder syndrome from childhood. Criteria for exclusion from electrical stimulation intervention include a history of epilepsy, migraine, the presence of metal implants, and other psychological disorders (other than Attention Deficit / Hyperactivity Disorder). The present study is applied and experimental type with multi-group pretest-posttest design in which one experimental group is exposed to transcranial electrical stimulation intervention and another experimental group is exposed to mindfulness-based cognitive therapy training. The control group did not receive any intervention. The statistical population of this study includes all people with attention deficit / hyperactivity disorder in Rasht. Five sessions of 20-minute transcranial electrical stimulation with a current of $1 \mathrm{~mA}$ were performed. Mindfulnessbased cognitive therapy interventions are based on the treatment program of Segal, Williams, and Teasdale(24). The program included 8, 90-minute sessions, and 45-minute homework three days a week. The post-test was held for all members of all three groups after the intervention sessions. A followup session was performed after three months to evaluate the durability of the intervention methods. Pre-test and post-test data analysis was performed by SPSS statistical calculation software version 25. This research was approved by Guilan University of Medical Sciences with ethics ID IR.GUMS.REC.1399.160 and can be viewed on the website of the National Ethics Committee website.

\section{2 measures}

Connor's Adult Attention Deficit / Hyperactivity Disorder Screening Scale: This diagnostic questionnaire is a tool with appropriate validity and reliability, including 26 items that are scored from zero to 3 and has five subscales. The subscales include Attention Deficit / Memory Problem, Restlessness / Hyperactivity Disorder, Impulsivity, Problems with Self-Esteem, and Attention Deficit/ Hyperactivity Disorder. The results of a differentiation analysis (25) showed that the sensitivity of this questionnaire with a cut-off point of 50 for the diagnosis of adult ADHD is $70 \%$ and its specificity is $99 \%$.

Go - No go: This test, the original version of which was designed by Hoffman in 1984, is used to measure inhibitory control and consists of two sets of stimuli. Subjects should respond to one group of stimuli (go) and refrain from responding to another group (no go). Because the number of go stimuli is usually greater than the number of non-go stimuli, the person is more willing to respond. The validity of this test is $0.87(26)$.

Strop test. The Strop test, or Strop effect, was introduced in 1935 by John Ridley Strop, one of the most widely used findings in cognitive psychology. One of the cognitive processes that is mostly considered in the implementation of this test is selective attention and this test evaluates the defect in this function(27). The reliability of this test is reported to be 0.88 (same source).

In order to analyze the data, in addition to descriptive methods (mean and standard deviation) for inferential analysis of test hypotheses, repeated measures analysis of variance was used. 


\section{3 tDCS intervention}

Direct currents generated by an electrical stimulator were applied through a pair of saline-soaked sponge electrodes $(5 \times 5 \mathrm{~cm})$. Bilateral DLPFC stimulation ( $1 \mathrm{~mA}, 20 \mathrm{~min}, 30 \mathrm{~s}$ ramp up, $30 \mathrm{~s}$ ramp down for active stimulation) was applied for 5 consecutive days with the anode electrode placed over the left DLPFC (F3), and the cathode electrode over the right DLPFC (F4) according to the 10-20 EEG International System.

\section{Results}

The results of Table 1 show that the mean of pre-test, post-test and follow-up of inhibitory control in the mindfulness group are $17.60,14.47$ and 14.40 with standard deviation of 1.35, 1.95 and 1.50, respectively. Also, the mean of pre-test, post-test and follow-up of inhibitory control in the electrical stimulation group are equal to $17.53,14.87$ and 15.47 with standard deviation of $1.40,1.80$ and 1.06, respectively. Based on the above table, it can be seen that the mean of pre-test, post-test and follow-up of inhibitory control in the control group are equal to $18.00,16.87$ and 17.20 with standard deviation of $1.89,1.52$ and 1.85 , respectively. Also, the mean of pre-test, post-test and follow-up of selective attention in the mindfulness group are $16,12.80$ and 11.60 with standard deviation of $1,0.86$ and 0.73 , respectively. Also, the mean of pre-test, post-test and follow-up of selective attention in the electrical stimulation group are equal to $15.67,13.07$ and 11.27 with standard deviation of $1.39,0.79$ and 0.88 , respectively. Based on the above table, it can be seen that the mean of pre-test, post-test and follow-up of selected attention in the control group are equal to $16.80,15.93$ and 15.53 with standard deviation of $1.61,1.22$ and 1.06 , respectively.

Table 1

Descriptive information of inhibitory control and selective attention

\begin{tabular}{|c|c|c|c|c|c|c|c|c|c|c|c|c|}
\hline groups & & $\begin{array}{l}\text { Pre- } \\
\text { test }\end{array}$ & & & & & Post-test & & & $\begin{array}{l}\text { Follow- } \\
\text { up }\end{array}$ & & \\
\hline $\begin{array}{l}\text { inhibitory } \\
\text { control }\end{array}$ & mean & SD & skewness & elongation & mean & SD & skewness & elongation & Mean & SD & skewness & elongation \\
\hline mINDFULNESS & 17.60 & 1.35 & 0.45 & -0.58 & 14.47 & 1.95 & -0.56 & -1.27 & 14.40 & 1.50 & -0.07 & -0.75 \\
\hline tDCS & 17.53 & 1.40 & 0.45 & -0.78 & 14.87 & 1.80 & -1.11 & 0.41 & 15.47 & 1.06 & -0.73 & 0.87 \\
\hline control & 18 & 1.89 & 0.44 & -0.65 & 16.87 & 1.52 & 0.65 & -0.39 & 17.20 & 1.85 & 0.28 & -0.71 \\
\hline $\begin{array}{l}\text { SELECTIVE } \\
\text { ATTENTION }\end{array}$ & Mean & SD & skewness & elongation & mean & SD & skewness & elongation & Mean & SD & skewness & elongation \\
\hline mindfulness & 16.00 & 1 & 0.49 & -0.91 & 12.80 & 0.86 & 0.43 & -1.54 & 11.60 & 0.73 & -0.39 & 0.42 \\
\hline tdcs & 15.67 & 1.39 & 1.23 & 1.22 & 13.07 & 0.79 & -0.12 & -1.34 & 11.27 & 0.88 & 0.11 & -0.48 \\
\hline control & 16.80 & 1.61 & 0.13 & 1.65 & 15.93 & 1.22 & -0.66 & -1.18 & 15.53 & 1.06 & 0.31 & -1.13 \\
\hline
\end{tabular}

The results of Table 2 show that the Levin test was used to investigate the assumption of equality of variances and considering the non-significance of Levin test, it can be concluded that the assumption of homogeneity of variance of error for research variables is established in all stages of research ( $p>$ 0.01).

Table 2

Levin test results, variance homogeneity of error

\begin{tabular}{llllll} 
variable & phase & $F$ & Df1 & Df2 & Significant level \\
\hline inhibitory control & Pretest & 0.75 & 2 & 42 & 0.47 \\
& Post-test & 1.06 & 2 & 42 & 0.35 \\
\hline & Follow-up & 2.10 & 2 & 42 & 0.13 \\
\hline selective attention & Pretest & 2.82 & 2 & 42 & 0.07 \\
& Post-test & 2.53 & 2 & 42 & 0.09 \\
\hline & Follow-up & 1.63 & 2 & 42 & 0.20
\end{tabular}

Table 3 shows that in the electrical stimulation group, post-test and follow-up scores of inhibitory control and selective attention, decreased compared to the pre-test $(P<0.01)$, while in the control group, there was not a significant difference in the three stages of the test $(P<0.05)$. Therefore, electrical stimulation therapy has been effective in improving inhibitory control, selective attention, working memory and clinical symptoms in the post-test and follow-up stages before the test. 
Table 3.

Analysis of repeated measures variance for mindfulness and tDCS groups

\begin{tabular}{|c|c|c|c|c|c|c|c|c|}
\hline \multirow[t]{3}{*}{ Variable } & & \multirow[t]{3}{*}{ time } & \multirow{3}{*}{ Mean difference } & \multicolumn{3}{|l|}{$\underline{\mathrm{tDCS}}$} & \multicolumn{2}{|c|}{ Control } \\
\hline & & & & SD & Significance & Mean difference & SD & Significance \\
\hline & & & & & Level & & & level \\
\hline inhibitory & Pre-test & Post-test & 2.66 & 0.67 & $p>0.001$ & 1.13 & 0.44 & $p>0.05$ \\
\hline control & & Follow-up & 2.06 & 0.43 & $p>0.001$ & 0.80 & 0.29 & $p>0.05$ \\
\hline & Post-test & Follow-up & -0.60 & 0.41 & $p>0.05$ & -0.33 & 0.46 & $p>0.05$ \\
\hline
\end{tabular}

\begin{tabular}{|c|c|c|c|c|c|c|c|c|}
\hline selective & Pre-test & Post-test & 11.53 & 0.66 & $p>0.01$ & -0.06 & 0.50 & $p>0.05$ \\
\hline attention & & Follow-up & 11.66 & 0.65 & $p>0.01$ & 0.06 & 0.44 & $p>0.05$ \\
\hline & Post-test & Follow-up & 0.13 & 0.35 & $p>0.05$ & 0.13 & 0.32 & $p>0.05$ \\
\hline
\end{tabular}

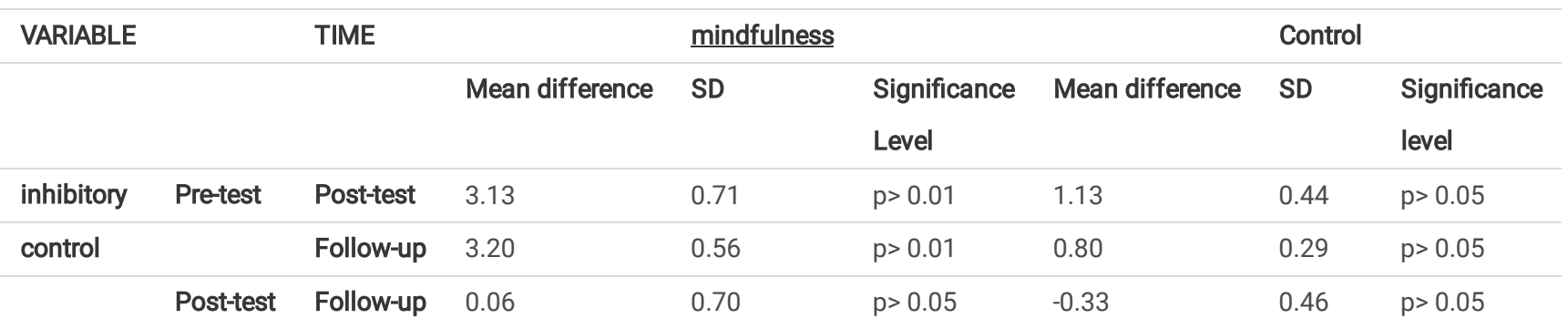

\begin{tabular}{rllllllll} 
selective & Pre-test & Post-test & 3.20 & 0.31 & $p>0.001$ & 0.86 & 0.49 & $p>0.05$ \\
atteention & & Follow-up & 4.40 & 0.21 & $p>0.001$ & 1.26 & 0.50 & $p>0.05$ \\
\cline { 3 - 4 } & Follow-up & 1.20 & 0.29 & $p>0.001$ & 0.40 & 0.33 & $p>0.05$
\end{tabular}

In addition, Table 3 shows that in the mindfulness group, post-test and follow-up scores of inhibitory control and selective attention decreased compared to the pre-test $(P<0.01)$, while in the control group there was no significant difference in the three stages of the test $(0.05)$. $<P)$. Therefore, mindfulness therapy has been effective in improving inhibitory control and selective attention in the post-test and follow-up stages to the pre-test.

Table 4 shows that the difference between the mean inhibitory control and selective attention between the groups of mindfulness and electrical stimulation is $-0.46,0.13$ and 0.33 with standard error of $0.39,0.27$ and 0.39 , respectively. Significant was not reported $(P<0.05)$ and it means that there is no significant difference between the two groups in terms of effectiveness on inhibitory control, selective attention and clinical symptoms and both groups were equally effective.

Table 4

The difference of effectiveness between two groups (mindfulness and tDCS)

\begin{tabular}{llllll} 
Variable & groups & & Mean difference & Standard error & Significance level \\
\hline inhibitory control & Mindfulness & tDCS & -0.46 & 0.39 & 0.73 \\
& & & & & \\
\hline selective attention & Mindfulness & tDCS & 0.13 & 0.27 & 0.99
\end{tabular}

\section{Discussion}

The results show that transcranial electrical stimulation has a significant effect on the formation and activity of the cerebral cortex. In this study, the electrical stimulation method used improved inhibitory control and the findings of this study in relation to this variable are in line with the findings of previous studies $(13,21)$. Anodic stimulation of the left lateral frontal cortex (the same area that is stimulated to improve attention) showed an 
improvement in attention efficiency. The findings of the present study are in line with the research results (16),(28) that show electrical stimulation of the brain is effective in improving the speed of attention processing. In fact, this stimulus method is a tool that increases the brain's ability to process input information. This feature increases the effectiveness of other treatments but does not eliminate the need for them. The present study showed that mindfulness-based cognitive therapy was effective in improving inhibitory control, selective attention and working memory in the post-test and follow-up stages of the pre-test. These results are in line with the results of research (9) and (22). Considering that cognitive and behavioral inhibition is one of the main sub-branches of executive functions and the results of neurological research also indicate the activity of the frontal cortex when performing tasks related to inhibitory control, and given that mindfulness exercises accelerate the activity of the frontal cortex (29), it is expected that improving the selfawareness of individuals, regardless of their ability to perform executive functions, will increase their ability to inhibit. The results of the present study are somewhat consistent with the results of the research (30),(29) that mindfulness training increases attention, self-control, mental flexibility and reduces behavioral problems, anxiety, impulsivity and hyperactivity. Attention regulation promotes awareness of cognitive and emotional experience as it happens moment by moment. Exercises designed to increase mindfulness usually include detailed attention training to maximize current awareness of the experience. According to the findings of this study, there is no significant difference between the two groups in terms of the effectiveness of intervention methods on inhibitory control, selective attention and clinical symptoms, and both groups were equally effective.

\subsection{Limitations}

The present study had limitations such as low sample size, available sampling method, sample selection from men, and not controlling the intelligence variable. Also, due to the long duration of the cognitive therapy of mindfulness, the fatigue of the learners can be seen. Due to the fact that the selected sample is related to the city of Rasht, so the generalization of the results to other parts of the country should be done with caution. In future research, it is suggested that the interventions in this study address other comorbid disorders with attention deficit / hyperactivity disorder in adolescence and youth, such as substance abuse prevention, and other disorders such as conduct disorder, anxiety, depression, and Investigate antisocial behavior.

\section{Conclusions}

It is worth noting that according to neuroimaging studies, structural changes occur in the forehead brain areas during mindfulness exercises. In fact, mindfulness itself can increase the cognitive control mechanisms of the forehead area (31). This raises the issue of overlap between the mechanism of action of intervention methods and the reinforcement of results. This means that mindfulness and transcranial electrical stimulation both strengthen the common areas of the frontal cortex. Therefore, the simultaneous effectiveness of these two methods on the studied variables can be due to the fact that common cortical regions are manipulated in both methods. The results and evidence of this study are contradictory to this issue in a study of (32) that states cross-sectional and short-term training is not possible to change the neural and biological structures, and vice versa, the method of electrical stimulation is recommended. Transcranial and mindfulness should be used by psychiatrists, psychologists and psychotherapists in psychiatric clinics and psychological service centers as a method of intervention and prevention.

\section{Declarations}

\section{Ethics approval and consent to participate}

The protocol was conducted in accordance with the latest version of the Declaration of Helsinki and was approved by the Institutional Review Board and ethical committee at the Guilan University of Medical Sciences. We obtained the written informed consent form the parents and oral assent from children before participation in the study. The ethical approval was obtained from the ethics committee review board in the Guilan University of Medical Sciences with ethics ID IR.GUMS.REC.1399.160 and can be viewed on the website of the National Ethics Committee website.

\section{Consent for publication}

Not applicable.

\section{Availability of data and materials}

The datasets generated and/or analyzed during the current study are not publicly available in order to maintain the anonymity of the participants. The data can be made available from the corresponding author on reasonable request.

\section{Declaration of Competing Interest}

All other declare no competing interests.

\section{Funding}

This research didn't receive any grant from any funding agency in the public, commercial or not-for-profit sectors. 


\section{Authors' contributions}

NM \& MN conceived the study. SMK \& MN collected the data and interpreted the data. AHK and SMK wrote the first draft. NM \& HS revised and reviewed. All authors read and approved the final manuscript.

\section{Acknowledgement}

The researchers announced their gratitude to all parents and children who participated in this study, as well as to the honorable authorities of Giulan Medical University, who are fully committed to this study.

\section{References}

1. Fayyad J, De Graaf R, Kessler R, Alonso J, Angermeyer M, Demyttenaere K, et al. Cross-national prevalence and correlates of adult attention-deficit hyperactivity disorder. The British Journal of Psychiatry. 2007;190(5):402-9.

2. Franke B, Faraone S, Asherson P, Buitelaar J, Bau C, Ramos-Quiroga JA, et al. The genetics of attention deficit/hyperactivity disorder in adults, a review. Molecular psychiatry. 2012;17(10):960-87.

3. Rubia K, Smith AB, Brammer MJ, Toone B, Taylor E. Abnormal brain activation during inhibition and error detection in medication-naive adolescents with ADHD. American Journal of Psychiatry. 2005;162(6):1067-75.

4. Smith AB, Taylor E, Brammer M, Toone B, Rubia K. Task-specific hypoactivation in prefrontal and temporoparietal brain regions during motor inhibition and task switching in medication-naive children and adolescents with attention deficit hyperactivity disorder. American Journal of Psychiatry. 2006;163(6):1044-51.

5. Nejati V, Salehinejad MA, Nitsche MA, Najian A, Javadi A-H. Transcranial direct current stimulation improves executive dysfunctions in ADHD: implications for inhibitory control, interference control, working memory, and cognitive flexibility. Journal of attention disorders. 2020;24(13):1928-43.

6. Fair DA, Bathula D, Nikolas MA, Nigg JT. Distinct neuropsychological subgroups in typically developing youth inform heterogeneity in children with ADHD. Proceedings of the National Academy of Sciences. 2012;109(17):6769-74.

7. Sonuga-Barke EJ. Causal models of attention-deficit/hyperactivity disorder: from common simple deficits to multiple developmental pathways. Biological psychiatry. 2005;57(11):1231-8.

8. Salehinejad MA, Nejati V, Mosayebi-Samani M, Mohammadi A, Wischnewski M, Kuo M-F, et al. Transcranial direct current stimulation in ADHD: a systematic review of efficacy, safety, and protocol-induced electrical field modeling results. Neuroscience Bulletin. 2020;36(10):1191-212.

9. Zylowska L, Ackerman DL, Yang MH, Futrell JL, Horton NL, Hale TS, et al. Mindfulness meditation training in adults and adolescents with ADHD: A feasibility study. Journal of attention disorders. 2008;11(6):737-46.

10. Hölzel BK, Carmody J, Vangel M, Congleton C, Yerramsetti SM, Gard T, et al. Mindfulness practice leads to increases in regional brain gray matter density. Psychiatry research: neuroimaging. 2011;191(1):36-43.

11. Lefaucheur J-P. A comprehensive database of published tDCS clinical trials (2005-2016). Neurophysiologie Clinique/Clinical Neurophysiology. 2016;46(6):319-98.

12. Nitsche MA, Cohen LG, Wassermann EM, Priori A, Lang N, Antal A, et al. Transcranial direct current stimulation: state of the art 2008. Brain stimulation. 2008;1(3):206-23.

13. Soltaninejad Z, Nejati V, Ekhtiari H. Effect of transcranial direct current stimulation on remediation of inhibitory control on right inferio frontal gyrus in attention deficit and hyperactivity symptoms. Rehabilitation Medicine. 2015;3(4).

14. Breitling C, Zaehle T, Dannhauer M, Bonath B, Tegelbeckers J, Flechtner $\mathrm{H}$, et al. Improving Interference control in ADHD patients with transcranial direct current stimulation (tDCS) Front. Cell Neurosci. 2016;10.

15. Salehinejad MA, Ghanavai E, Rostami R, Nejati V. Cognitive control dysfunction in emotion dysregulation and psychopathology of major depression (MD): Evidence from transcranial brain stimulation of the dorsolateral prefrontal cortex (DLPFC). Journal of affective disorders. 2017;210:241-8.

16. Bandeira ID, Guimarães RSQ, Jagersbacher JG, Barretto TL, de Jesus-Silva JR, Santos SN, et al. Transcranial direct current stimulation in children and adolescents with attention-deficit/hyperactivity disorder (ADHD) a pilot study. Journal of child neurology. 2016;31(7):918-24

17. Cruz Gonzalez P, Fong KN, Brown T. The effects of transcranial direct current stimulation on the cognitive functions in older adults with mild cognitive impairment: a pilot study. Behavioural neurology. 2018;2018.

18. Rahmani J, Ajilchi B, Zareian E. Relationship between mindfulness and cognitive abilities in blind veteran athletes. Iranian Journal of War and Public Health. 2017;9(2):105-10.

19. Shapiro SL, Brown KW, Biegel GM. Teaching self-care to caregivers: Effects of mindfulness-based stress reduction on the mental health of therapists in training. Training and education in professional psychology. 2007;1(2):105.

20. Nejati V, Zabihzadeh A, Nikfarjam M. The relationship of mindfulness with sustained and selective attentional performance. Research in Cognitive and Behavioral Sciences. 2012;2(2):31-42.

21. Cachoeira CT, Leffa DT, Mittelstadt SD, Mendes LST, Brunoni AR, Pinto JV, et al. Positive effects of transcranial direct current stimulation in adult patients with attention-deficit/hyperactivity disorder A pilot randomized controlled study. Psychiatry Research. 2017;247:28-32. 
22. Clavenna A, Bonati M. Safety of medicines used for ADHD in children: a review of published prospective clinical trials. Archives of disease in childhood. 2014;99(9):866-72.

23. Shecter C. Mindfulness training for adolescents with ADHD and their families: A time-series evaluation: University of Toronto; 2013.

24. Segal ZV, Teasdale JD, Williams JMG. Mindfulness-Based Cognitive Therapy: Theoretical Rationale and Empirical Status. 2004.

25. Mokhtari H, Rabiei M, Salimi SH. Psychometric properties of the persian version of adult attention-deficit/hyperactivity disorder self-report scale. Iranian Journal of Psychiatry and Clinical Psychology. 2015;21(3):244-53.

26. Ramesh S, Samimi Z, Mashhadi A. The Improvement Cognitive Inhibition in Children with Attention Deficit/Hyperactivity Disorder in the Context of Emotional Working Memory Training. Journal of Cognitive Psychology. 2018;6(1):31-40.

27. Narimani M, Pouresmali A, Andalib Kouraeim M, Aghajanei S. A comparison of Stroop performance in students with learning disorder and normal students. Journal of Learning Disabilities. 2012;2(1):138-58.

28. Gonzalez PC, Fong KN, Brown T. Transcranial direct current stimulation as an adjunct to cognitive training for older adults with mild cognitive impairment: A randomized controlled trial. Annals of physical and rehabilitation medicine. 2021;64(5):101536.

29. Semple RJ. Mindfulness-based cognitive therapy for children: A randomized group psychotherapy trial developed to enhance attention and reduce anxiety: Columbia University; 2005.

30. Mitchell JT, Zylowska L, Kollins SH. Mindfulness meditation training for attention-deficit/hyperactivity disorder in adulthood: Current empirical support, treatment overview, and future directions. Cognitive and behavioral practice. 2015;22(2):172-91.

31. Afi E, Esteki M, Madahi M, Hasani F. Comparison of the effectiveness of mindfulness-based cognative therapy and the use of neurofeedback on emotional self regulation in children with ADHD. Journal of diability studies. 2020;75(10):1-9.

32. Sadat Karimi L, Zare H, Hadianfard H. Effects of music therapy on selective attention of children with attention deficit-hyperactivity disorder. Iranian journal of exceptional children. 2011;1:23-34. 